\title{
Numerical solution of Schrödinger equation for biphoton wave function in twisted waveguide arrays
}

\author{
D. N. Vavulin ${ }^{1}$, A. A. Sukhorukov ${ }^{2}$ \\ ${ }^{1}$ ITMO University, St. Petersburg, Russia \\ ${ }^{2}$ Nonlinear Physics Centre, Research School of Physics and Engineering, \\ Australian National University, Canberra, ACT 2601, Australia \\ dima-vavulin@mail.ru, Andrey.Sukhorukov@anu.edu.au
}

PACS 42.50.Tx, 42.65.Wi, 42.65.Lm DOI 10.17586/2220-8054-2015-6-5-689-696

We consider the generation of entangled biphoton states with orbital-angular-momentum in triangular quadratic waveguide arrays with twisted geometry. For this purpose, we derive the Shrödinger equation for biphoton wave function and equation for pump field profile. We describe numerically the process of biphoton generation through spontaneous four-wave mixing. We suggest that biphoton correlations can be controlled by the amount of twist and pump field profile.

Keywords: entangled states, orbital-angular-momentum, optical vortices, twisted waveguides, spontaneous four-wave mixing.

Received: 30 June 2015

Revised: 5 August 2015

\section{Introduction}

Quantum biphoton states with orbital-angular-momentum (OAM) can be used in quantum communications to extend the alphabet of quantum cryptography, which can increase the transmission rate [7]. Of particular interest are quantum walks, where the interference of several walkers can be used to realize various simulations, including database search [1,12], quantum teleportation, and quantum cryptography [2-4], enabling absolutely secure communications. Quantum walks and generation of biphoton states with OAM can be implemented in arrays of coupled waveguides with closed-loop boundary conditions [5]. Recently, a new type of coupled waveguide with a twisted geometry was demonstrated in a photonic-crystal fiber [6]. Such waveguides are able to effectively provide the transfer of OAM states. The behavior and generation of biphotons in twisted waveguides can be described by the Shrödinger equation and evolution of the pump profile can be desribed by the classical coupled-mode equations. The biphoton entanglement with OAM was first experimentally demonstrated by Zeilinger et al. using the process of spontaneous parametric down-conversion (SPDC) [7]. We consider a linear polarization of light and small twist of waveguides, with the amount of twist $\lesssim 1^{\circ}$, and in this case the structure of the pump and biphoton modes is essentially the same as in the straight array. We formulate the mathematical model under such conditions.

In our work, we consider generation of biphonons through spontaneous four-wave mixing (SFWM) through $\chi^{(3)}$ nonlinearity in optical fibers. We have not considered $\chi^{(2)}$ nonlinearity, because it requires a crystal lattice without a center of inversion and it is difficult to realize in the optical fiber, unlike $\chi^{(3)}$ nonlinearity which occurs in conventional optical fibers. However, in the case of $\chi^{(3)}$ nonlinearity, the frequencies of pump wave 
and biphotons may be close to each other, which requires narrow-band filtering before the single-photon detectors [15].

We numerically solve the classical coupled-mode equations for pump profile and Shrödinger equation for biphotons in twisted waveguides.

\section{Derivation of Schrödinger equation for biphoton wave function in a twisted waveguide array}

The normalized pump field profile evolution along the propagation distance $z$ is defined through the classical coupled-mode equations [8]:

$$
i \frac{d E_{n}}{d z}+C(\omega) \exp \left[-i \omega \dot{\chi_{0}}(z)\right] E_{n+1}+C(\omega) \exp \left[i \omega \dot{\chi_{0}}(z)\right] E_{n-1}=0
$$

where $E_{n}$ is the complex field amplitude in the nth waveguide, and $E_{N}$ maps to $E_{0}$, and $E_{-1}$ maps to $E_{N-1}$ due to closed-loop boundary conditions [9], $n$ is the waveguide number, $N$ is the total number of waveguides, $\chi_{0}(z) \equiv \chi_{0}(z+L)$ is the periodic waveguide bending profile, $L$ is the modulation period, coefficient $C(\omega)$ defines a coupling strength between the neighboring waveguides (it characterizes diffraction strength in a straight waveguide array (WGA) with $\chi_{0} \equiv 0$ ) [10]. In our case, $\dot{\chi}_{0}(z)=$ const, because the waveguide bending profile is the same for all values of $z$.

The eigenmode solutions of Eqs. (1) are $E_{n(z)}^{(m)}=\varepsilon_{n}^{(m)} \exp \left(i \beta_{m} z\right)$, where $m=0, \ldots, N-1$ is the mode number, $\varepsilon_{n}^{(m)}=\exp (i 2 \pi m n / N)$ are the mode profiles, and $\beta_{m}=2 C \cos \left(2 \pi m / N-\phi_{0}\right)$ (where $\left.\phi_{0}=\omega \dot{\chi}_{0}(z)\right)$ are the propagation constants. The eigenmodes $E_{n(z)}^{(m)}$ represent discrete optical vortices [14], or states with OAM due to a twisted structure of their phase profiles. Here, the supermode number $m$ corresponds to the OAM.

Generation of photon pairs in cubic nonlinear WGAs through SFWM in the absence of multiple photon pairs can be characterized by the evolution of a bi-photon wave function $\psi_{n_{s}, n_{i}}(z)$ in a Schrödinger-type equation. The equation is obtained from the Hamiltonian, and has a form similar to that of quadratic media [13]:

$$
\begin{aligned}
& i \frac{d \psi_{n_{s}, n_{i}}(z)}{d z}= \\
& \quad \begin{aligned}
-C\left[\exp \left[i \omega \dot{\chi_{0}}(z)\right] \psi_{n_{s-1}, n_{i}}(z)+\exp \left[i \omega \dot{\chi_{0}}(z)\right] \psi_{n_{s}, n_{i-1}}(z)+\exp \left[-i \omega \dot{\chi}_{0}(z)\right] \psi_{n_{s+1}, n_{i}}(z)\right. \\
\left.\quad+\exp \left[-i \omega \dot{\chi}_{0}(z)\right] \psi_{n_{s}, n_{i+1}}(z)\right]-\Delta \beta^{(0)} \psi_{n_{s}, n_{i}}(z)+i \gamma E_{n_{s}}^{(p)}(z) E_{n_{s}}^{(p)}(z) \delta_{n_{s}, n_{i}}
\end{aligned}
\end{aligned}
$$

where $n_{s}$ and $n_{i}$ are the waveguide numbers describing the positions of the signal and the idler photons, and $E_{n_{s}}^{(p)}(z)$ is the pump amplitude in waveguide number $n_{s} . \Delta \beta^{(0)}$ is the linear four-wave mixing phase-mismatch in a single waveguide, $\gamma$ is a nonlinear coefficient [11]. Dimensionless quantities $\Delta \beta^{(0)}$ and $\gamma$ in the numerical simulations were considered equal to 1 . 
It is convenient to make a transformation $\psi_{n_{s}, n_{i}}(z)=\psi_{n_{s}, n_{i}}(z) \exp \left(-i \Delta \beta^{(0)} z\right)$. Then, (2) takes the form:

$$
\begin{aligned}
& i \frac{d \psi_{n_{s}, n_{i}}(z)}{d z}= \\
& \quad-C\left[\exp \left[i \omega \dot{\chi_{0}}(z)\right] \psi_{n_{s-1}, n_{i}}(z)+\exp \left[i \omega \dot{\chi_{0}}(z)\right] \psi_{n_{s}, n_{i-1}}(z)+\exp \left[-i \omega \dot{\chi_{0}}(z)\right] \psi_{n_{s+1}, n_{i}}(z)\right. \\
& \left.\quad+\exp \left[-i \omega \dot{\chi_{0}}(z)\right] \psi_{n_{s}, n_{i+1}}(z)\right]+i \gamma E_{n_{s}}^{(p)}(z) E_{n_{s}}^{(p)}(z) \delta_{n_{s}, n_{i}} \exp \left(i \Delta \beta^{(0)} z\right) .
\end{aligned}
$$

\section{Numerical solution}

\subsection{Numerical solution for pump field}

We consider the algorithm for solving equation (1) for a system of three waveguides with closed-loop boundary conditions. Schematically, the system appears as shown in Fig. 1.

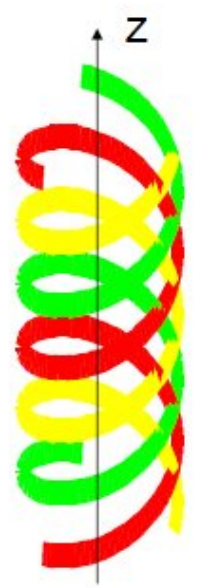

FIG. 1. Scheme of coupled twisted waveguides

Firstly, we consider a system with open boundary conditions, which is described by a three-diagonal matrix of the form:

$$
\left(\begin{array}{ccc}
B_{1} & C_{2} & 0 \\
A_{2} & B_{2} & C_{2} \\
0 & A_{3} & B_{3}
\end{array}\right)\left(\begin{array}{c}
E_{1} \\
E_{2} \\
E_{3}
\end{array}\right)=\left(\begin{array}{c}
D_{1} \\
D_{2} \\
D_{3}
\end{array}\right),
$$

where $A_{n}=-i(\Delta z / 2) C \exp \left[i \omega \dot{\chi}_{0}(z)\right] ; \quad B_{n}=1 ; \quad C_{n}=-i(\Delta z / 2) C \exp \left[-i \omega \dot{\chi}_{0}(z)\right]$; $E_{n}=E_{n}(z) ; D_{n}=-A_{n} E_{n-1}(z-\Delta z)+B_{n} E_{n}(z-\Delta z)-C_{n} E_{n+1}(z-\Delta z) ; n=1,2, \ldots, N$; $\Delta z$ - step along $z$ axis.

This matrix ratio is equivalent to the system of linear equations of the form $A E=D$, where $E$ is a column vector with unknown values, and the matrix $A$ and a column vector $D$ are known. The solution can be found by the shuttle method or, for example, by the formula $E=A^{(-1)} D=\operatorname{linsolve}(A, D)$, which is implemented in a software package Matlab. relation:

In the case of closed-loop boundary conditions, we obtain the following matrix 


$$
\left(\begin{array}{lll}
B_{1} & C_{2} & A_{1} \\
A_{2} & B_{2} & C_{2} \\
C_{3} & A_{3} & B_{3}
\end{array}\right)\left(\begin{array}{l}
E_{1} \\
E_{2} \\
E_{3}
\end{array}\right)=\left(\begin{array}{c}
D_{1} \\
D_{2} \\
D_{3}
\end{array}\right)
$$

The solution can also be found by the formula $E=A^{(-1)} D=\operatorname{linsolve}(A, D)$. For clarity, we give the matrix ratio for the system of five waveguides, which is constructed by a similar algorithm:

$$
\left(\begin{array}{ccccc}
B_{1} & C_{1} & 0 & 0 & A_{1} \\
A_{2} & B_{2} & C_{2} & 0 & 0 \\
0 & A_{3} & B_{3} & C_{3} & 0 \\
0 & 0 & A_{4} & B_{4} & C_{4} \\
C_{5} & 0 & 0 & A_{5} & B_{5}
\end{array}\right)\left(\begin{array}{l}
E_{1} \\
E_{2} \\
E_{3} \\
E_{4} \\
E_{5}
\end{array}\right)=\left(\begin{array}{c}
D_{1} \\
D_{2} \\
D_{3} \\
D_{4} \\
D_{5}
\end{array}\right) .
$$

Thus, we can similarly obtain the numerical solution for the system of any number of waveguides. The figures show the numerical solutions for the direct (Fig. 2) and twisted (Fig. 3) arrays of the three waveguides.

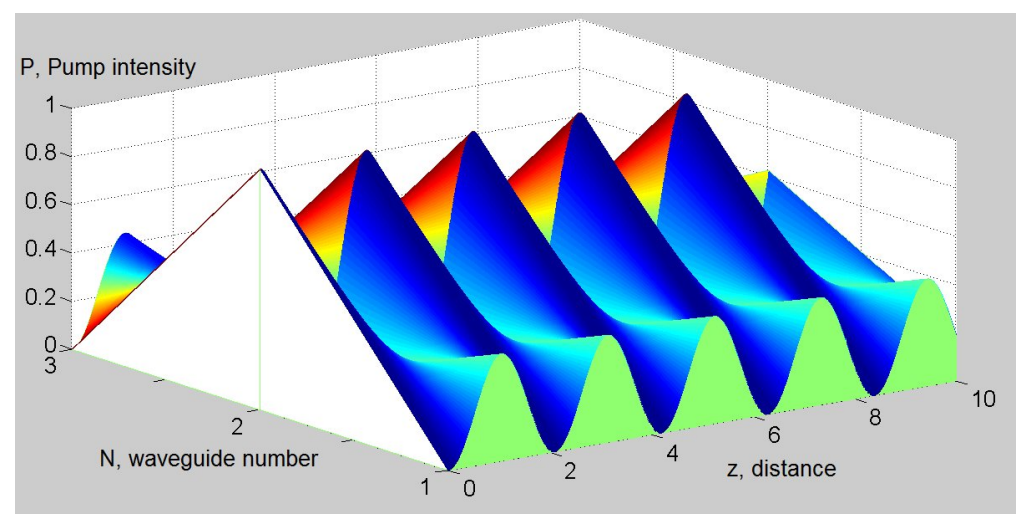

FIG. 2. Pump power $\left(\left|E_{n}\right|^{2}\right)$, in the system of three straight waveguides

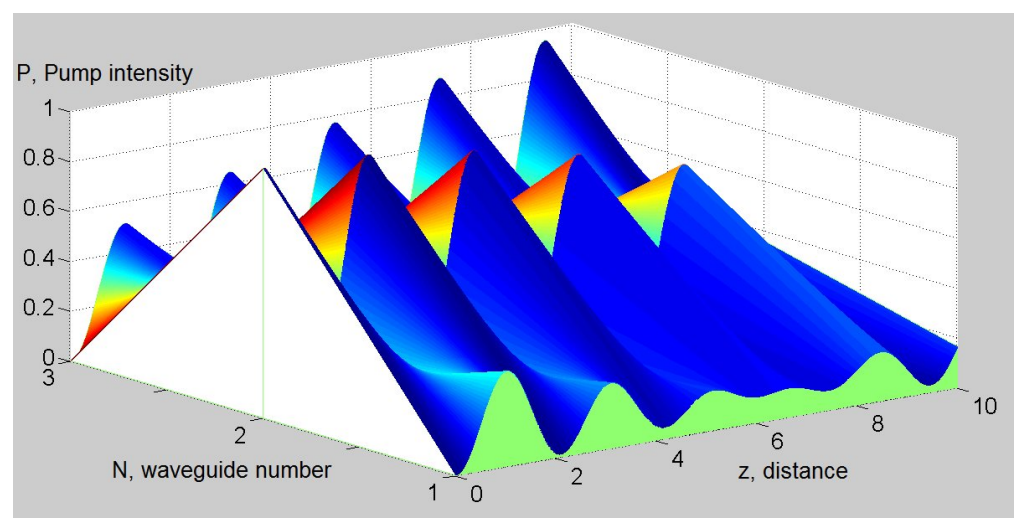

FIG. 3. Pump power $\left(\left|E_{n}\right|^{2}\right)$, in the system of three twisted waveguides 
Numerical solution of Schrödinger equation for biphoton wave function...

\subsection{Numerical solution of Schrödinger equation for biphoton wave function}

Now, we consider the numerical algorithm for Schrödinger equation (3) for system of three twisted waveguides with closed boundary conditions.

We transform this equation by the Crank-Nicolson scheme to the following form:

$$
\begin{aligned}
i \psi_{n_{s}, n_{i}}^{j+1} & =i \psi_{n_{s}, n_{i}}^{j}-\frac{\Delta z}{2}\left[\alpha_{2}\left(\psi_{n_{s-1}, n_{i}}^{j}+\psi_{n_{s}, n_{i-1}}^{j}\right)+\alpha_{1}\left(\psi_{n_{s+1}, n_{i}}^{j}+\psi_{n_{s}, n_{i+1}}^{j}\right)\right. \\
& \left.+\alpha_{2}\left(\psi_{n_{s-1}, n_{i}}^{j+1}+\psi_{n_{s}, n_{i-1}}^{j+1}\right)+\alpha_{1}\left(\psi_{n_{s+1}, n_{i}}^{j+1}+\psi_{n_{s}, n_{i+1}}^{j+1}\right)\right] \\
& +\frac{\Delta z}{2} i \gamma\left(E_{n_{s}}^{j}\right)^{2} \delta_{n_{s}, n_{i}} \exp \left(i \Delta \beta^{(0)} \Delta z j\right)+\frac{\Delta z}{2} i \gamma\left(E_{n_{s}}^{j+1}\right)^{2} \delta_{n_{s}, n_{i}} \exp \left(i \Delta \beta^{(0)} \Delta z(j+1)\right),
\end{aligned}
$$

where $\alpha_{1}=C \exp \left[-i \omega \dot{\chi_{0}}\right], \alpha_{2}=C \exp \left[i \omega \dot{\chi_{0}}\right]$.

Furthermore:

$$
\begin{aligned}
& -r_{2} \cdot \psi_{n_{s}-1, n_{i}}^{j+1}-r_{2} \cdot \psi_{n_{s}, n_{i}-1}^{j+1}+\psi_{n_{s}, n_{i}}^{j+1}-r_{1} \cdot \psi_{n_{s}+1, n_{i}}^{j+1}-r_{1} \cdot \psi_{n_{s}, n_{i}+1}^{j+1} \\
& =r_{2} \cdot \psi_{n_{s}-1, n_{i}}^{j}+r_{2} \cdot \psi_{n_{s}, n_{i}-1}^{j}+\psi_{n_{s}, n_{i}}^{j}+r_{1} \cdot \psi_{n_{s}+1, n_{i}}^{j}+r_{1} \cdot \psi_{n_{s}, n_{i}+1}^{j} \\
& +r_{3}\left(E_{n_{s}}^{j}\right)^{2} \exp \left(r_{4} j\right)+r_{3}\left(E_{n_{s}}^{j+1}\right)^{2} \exp \left(r_{4}(j+1)\right),
\end{aligned}
$$

where $r_{1}=i \frac{\Delta z}{2} \alpha_{1}, r_{2}=i \frac{\Delta z}{2} \alpha_{2}, r_{3}=\frac{\Delta z}{2} \gamma, r_{4}=i \Delta \beta^{(0)} \Delta z$.

Thus, we obtain the following numerical scheme:

$$
-r_{2} \cdot \psi_{n_{s}-1, n_{i}}^{j+1}-r_{2} \cdot \psi_{n_{s}, n_{i}-1}^{j+1}+\psi_{n_{s}, n_{i}}^{j+1}-r_{1} \cdot \psi_{n_{s}+1, n_{i}}^{j+1}-r_{1} \cdot \psi_{n_{s}, n_{i}+1}^{j+1}=D_{n_{s}, n_{i}}^{j},
$$

where

$$
\begin{aligned}
D_{n_{s}, n_{i}} & =r_{2} \cdot \psi_{n_{s}-1, n_{i}}^{j}+r_{2} \cdot \psi_{n_{s}, n_{i}-1}^{j}+\psi_{n_{s}, n_{i}}^{j}+r_{1} \cdot \psi_{n_{s}+1, n_{i}}^{j}+r_{1} \cdot \psi_{n_{s}, n_{i}+1}^{j} \\
& +r_{3}\left(E_{n_{s}}^{j}\right)^{2} \exp \left(r_{4} j\right)+r_{3}\left(E_{n_{s}}^{j+1}\right)^{2} \exp \left(r_{4}(j+1)\right) .
\end{aligned}
$$

As a result, we now have a two-dimensional problem. Next, we can obtain the one-dimensional problem by reshaping matrix for the psi-function in a column vector:

$$
\left(\begin{array}{ccc}
\psi_{1,1} & \psi_{1,2} & \psi_{1,3} \\
\psi_{2,1} & \psi_{2,2} & \psi_{2,3} \\
\psi_{3,1} & \psi_{3,2} & \psi_{3,3}
\end{array}\right) \longrightarrow\left(\begin{array}{c}
\psi_{1,1} \\
\psi_{1,2} \\
\psi_{1,3} \\
\psi_{2,1} \\
\psi_{2,2} \\
\psi_{2,3} \\
\psi_{3,1} \\
\psi_{3,2} \\
\psi_{3,3}
\end{array}\right)
$$

Finally, we have a five-diagonal matrix. In matrix form, the problem takes the following form: 


$$
\left(\begin{array}{ccccccccc}
1 & -r_{1} & -r_{2} & -r_{1} & 0 & 0 & -r_{2} & 0 & 0 \\
-r_{2} & 1 & -r_{1} & 0 & -r_{1} & 0 & 0 & -r_{2} & 0 \\
-r_{1} & -r_{2} & 1 & 0 & 0 & -r_{1} & 0 & 0 & -r_{2} \\
-r_{2} & 0 & 0 & 1 & -r_{1} & -r_{2} & -r_{1} & 0 & 0 \\
0 & -r_{2} & 0 & -r_{2} & 1 & -r_{1} & 0 & -r_{1} & 0 \\
0 & 0 & -r_{2} & -r_{1} & -r_{2} & 1 & 0 & 0 & -r_{1} \\
-r_{1} & 0 & 0 & -r_{2} & 0 & 0 & 1 & -r_{1} & -r_{2} \\
0 & -r_{1} & 0 & 0 & -r_{2} & 0 & -r_{2} & 1 & -r_{1} \\
0 & 0 & -r_{1} & 0 & 0 & -r_{2} & -r_{1} & -r_{2} & 1
\end{array}\right)\left(\begin{array}{l}
\psi_{1,1} \\
\psi_{1,2} \\
\psi_{1,3} \\
\psi_{2,1} \\
\psi_{2,2} \\
\psi_{2,3} \\
\psi_{3,1} \\
\psi_{3,2} \\
\psi_{3,3}
\end{array}\right)=\left(\begin{array}{l}
D_{1,1} \\
D_{1,2} \\
D_{1,3} \\
D_{2,1} \\
D_{2,2} \\
D_{2,3} \\
D_{3,1} \\
D_{3,2} \\
D_{3,3}
\end{array}\right) .
$$

This matrix ratio is equivalent to the matrix system of linear equations of the form $A \Psi=D$, where $\Psi$ is a vector-column with unknown values, and the matrix $A$ and a column vector $D$ are known. The solution can be found by splitting methods or, for example, by the formula $\Psi=A^{(-1)} D=\operatorname{linsolve}(A, D)$, implemented in the Matlab software package.

Figures 4 and 5 show the numerical solutions for the squared modulus of the biphoton wave function respectively for forward and twisted arrays of three waveguides. In Figs. 4 and 5, we see 9 imaginary waveguides, which corresponds to the 3 real waveguides. Thus, waveguides with numbers 1-3 correspond to the biphoton wave function with signal photon located in the 1st wavegude and idler photon located in 1-3 waveguides respectively. Similarly, waveguides 4-6 correspond to the signal photon located in the 2 nd waveguide and the idler photon located in waveguides 1-3, and waveguides 7-9 correspond to the signal photon located in the 3rd waveguide and the idler photon located in waveguides $1-3$.

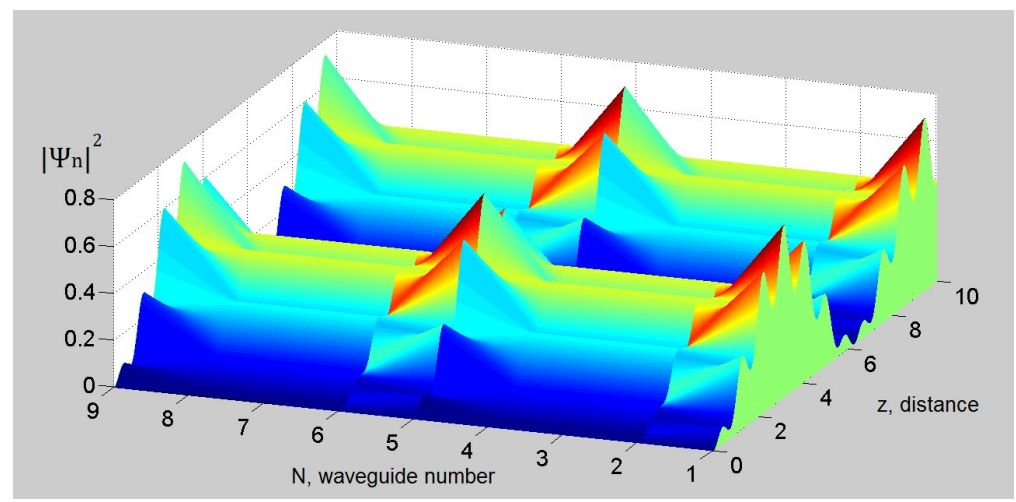

FIG. 4. Two-photon correlations $\left(\left|\Psi_{n_{s}, n_{i}}\right|^{2}\right)$, in case of photon generation by SFWM from a pump inside the array for system of three straight waveguides

If we transform the Fig. 5 to a more convenient form Fig. 6, we can see the twophoton correlations $\left(\left|\Psi_{n_{s}, n_{i}}\right|^{2}\right)$ at three different distances, which are of practical interest (a: $z=0 ; \mathrm{b}: z=0.375 L$; c: $z=0.604 L$, where $L$ is the distance at which the biphoton wave function restores its initial state, when photons propagate in the regime of quantum walks in the absence of pumping). At $z=0$ (a), there are no photons. At distance (b), the generated photons are mostly bunched: appearing in the same waveguides. In (c), the photons are anti-bunched; appearing at different waveguides. 


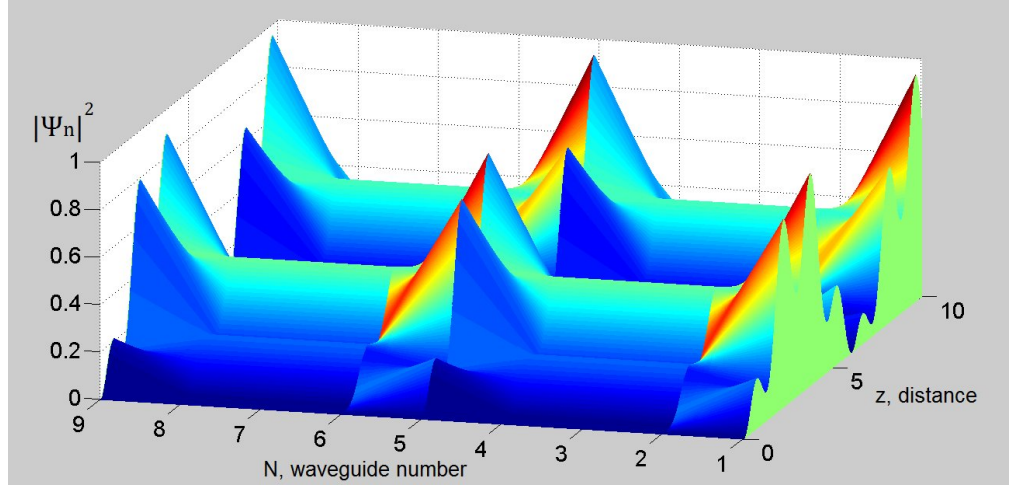

FIG. 5. Two-photon correlations $\left(\left|\Psi_{n_{s}, n_{i}}\right|^{2}\right)$, in case of photon generation by SFWM from a pump inside the array for system of three twisted waveguides
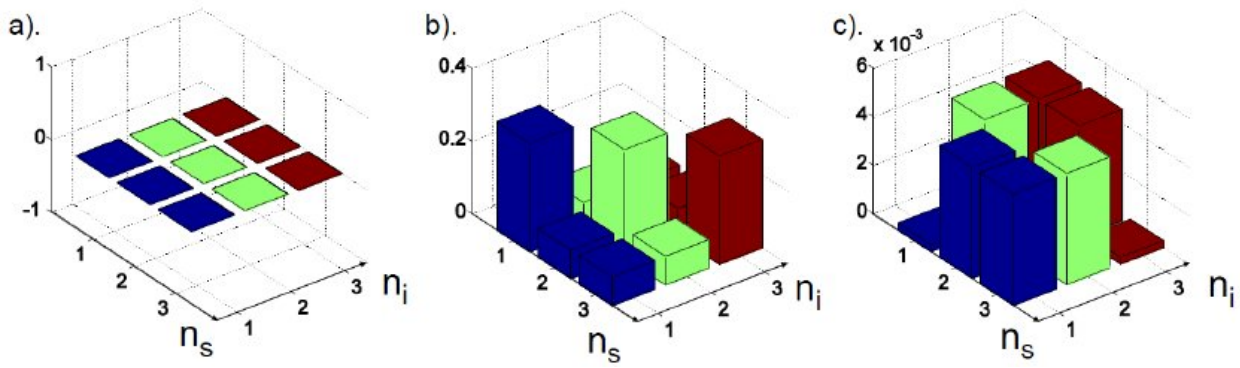

FIG. 6. Two-photon correlations $\left(\left|\Psi_{n_{s}, n_{i}}\right|^{2}\right)$, in case of photon generation by SFWM from a pump inside the array. (a) $z=0$, (b) $z=0.375 \mathrm{~L}$, (c) $z=0.604 L$. At $z=0$ (a), there are no photons. At distance (b), the generated photons are mostly bunched; appearing in the same waveguides. In (c), the photons are anti-bunched.

Thus, we obtain that in the case of photon generation by SFWM from a pump inside the array, the signal and idler photons can be bunched at some distances and anti-bunched at other distances.

\section{Conclusion}

We have numerically solved the classical equation of coupled modes for the pump wave and the Schrödinger equation for biphoton wave function of three twisted waveguides. We analyzed integrated photon generation through spontaneous four-wave mixing. We have shown that the generated photons can demonstrate bunching behavior at some distances and anti-bunching behavior at other distances. These features can be controlled by the amount of twist and the input pump profile. Also, in the future, it is of interest to consider the effects of nonlinear phase modulation and self-focusing due to the pumping.

\section{Acknowledgements}

Authors wishing to acknowledge the Dynasty foundation for their financial support. 


\section{References}

[1] Hamilton C.S., Kruse R., et al. Driven Quantum Walks. Phys. Rev. Lett., 2014, 113, P. 083602-5.

[2] Ekert A.K., Rarity J.G., Tapster P.R., Palma G.M. Practical quantum cryptography based on two-photon interferometry. Phys. Rev. Lett., 1992, 69, P. 1293.

[3] Egorov V.I., Vavulin D.N., et al. Analysis of a sidebands based quantum cryptography system with different detector types. Nanosystems: Phys., Chem., Math., 2013, 4 (2), P. 190-195.

[4] Vavulin D.N., Egorov V.I., Gleim A.V., Chivilikhin S.A. Determining influence of fourwave mixing effect on quantum key distribution. Journal of Physics: Conference Series, 2014, 541, P. 012066.

[5] Owens J.O. et al. Two-photon quantum walks in an elliptical direct-write waveguide array. New $J$. Phys., 2011, 13, P. 075003-13.

[6] Xi X.M., Wong G.K.L., et al. Orbital-angular-momentum-preserving helical Bloch modes in twisted photonic crystal fiber. Optica, 2014, 1 (3), P. 165-169.

[7] Mair A., Vaziri A., Weihs G., Zeilinger A. Entanglement of the orbital angular momentum states of photons. Nature, 2001, 412, P. 313.

[8] Christodoulides D.N., Lederer F., Silberberg Y.. Discretizing light behaviour in linear and nonlinear waveguide lattices. Nature, 2003, 424, P. 817-823.

[9] Markin D.M., Solntsev A.S., Sukhorukov A.A. Generation of orbital-angular-momentum-entangled biphotons in triangular quadratic waveguide arrays. Phys. Rev. A, 2013, 87, P. 063814-5.

[10] Garanovich I.L., Longhi S., Sukhorukov A.A., Kivshar Yu.S. Light propagation and localization in modulated photonic lattices and waveguides. Phys. Rep., 2012, 518, P. 1-79.

[11] Solntsev A.S., Sukhorukov A.A., Neshev D.N., Kivshar Yu.S. Photon-pair generation in arrays of cubic nonlinear waveguides. Optics Express, 2012, 20 (24), P. 27441.

[12] Jonathan C.F., Thompson M. and Thompson M.G. Quantum optics: An entangled walk of photons. Nature, 2012, 484, P. 47-48.

[13] Grafe M., Solntsev A.S., et al. Biphoton generation in quadratic waveguide arrays: A classical optical simulation. Scientific Reports, 2012, 2 (562), P. 1-5.

[14] Desyatnikov A.S., Dennis M.R., Ferrando A. All-optical discrete vortex switch. Phys. Rev. A, 2011, 83, P. 063822.

[15] Agrawal G. Applications of Nonlinear Fiber Optics. San Diego, Academic Press, 2001, 472 p. 